\title{
Optimization A New Mathematical Model of Hybrid Flow Shop Work Order Problem By The Genetic Algorithm
}

\author{
H.Asadi*, N. Shahsavari Pour, M.Pour kheradmand \\ *Department of Industrial Engineering, Science and Research Branch, Islamic \\ Azad University, Kerman, Iran \\ E-mail:hamed_assadi2000@yahoo.com \\ Department of Industrial Engineering, Science and Research Branch, Islamic \\ Azad University, Kerman, Iran \\ E-mail: shahsavari_n@alum.sharif.edu \\ Department of Industrial Engineering, Science and Research Branch, Islamic \\ Azad University, Kerman, Iran \\ E-mail:mansour_kheradmand@yahoo.com
}

\begin{abstract}
In competitive business, one of the challenges of management in industrial units is reducing the prime cost. Hybrid flow shop is one of the common production environments which lead to a significant decrease in production costs if it has a good and appropriate scheduling in production. Hybrid flow-shop problems overcome one of the limitations of the classical flow-shop model by allowing parallel processors at each stage of task Processing. In this paper we study the hybrid flow shop work order problems. A brief enumeration of the essential constraints that characterize this kind of organization is given. Problem is minimizing the production and inventory cost in Hybrid flow-shop organization. To solve problem we used the genetic algorithm to obtain the minimum of production cost. An illustrative example explains in detail the feature of the proposed model.
\end{abstract}

Keywords: hybrid flow shop, mathematical model, production cost,inventory cost, genetic algorithm. 


\section{Introduction}

One of the managers' problems is reduce production costs in the management of manufacturing companies. Hybrid Flow Shops (HFS) is common manufacturing environments in which a set of $\mathrm{n}$ jobs are to be processed in a series of $\mathrm{w}$ stages. There are two important questions that should be considered in scheduling, including. First, when can we produce a product? and Second, How much is produce? The first question is in relation with production scheduling problems.Most research focus on scheduling problems. So far, many literatures are available for scheduling problems with limited buffers. Hall and Sriskan darajah N. G. Hall, C. Sriskan darajah [9] provided a survey for scheduling problems with blocking and no-wait in process. M. S. Salvador[10] first considered hybrid flow shop with no buffers between stages. He applied branchand-bound techniques to minimize makespan. C. Rajendran, D. Chaudhuri[11]utilized branch-and-bound but they also focus only onpermutation schedules. S. A. Brah, J. L. Hunsucker[12] used branch-and-bound in the hybrid flow shop with an arbitrary number of stages and intermediate buffers. Moreover, they provide a means by which nonpermutation schedules or schedules with inserted idle time can be created.T. Sawik [15]presented a mixed integer programming formulation for scheduling flexibleflow line with limited buffers and also a FFS with limited intermediate buffers T. Sawik [13] and with noprocess buffers.T. Sawik[14]. Torabi, B. Karimi [7]proposed two meta-heuristic algorithms, including the HGA and simulated annealing (SA), to solve a new 0 1mixed-nonlinear mathematical model of the economic lot-sizing and scheduling problem in flexible flow lines with unrelated parallel processors over a finite planning horizon. The objective determines a cyclic schedule by minimizing the sum of setup and inventory holding costs per unit time without any stock-out. Akrami et al. B. Akrami, B. Karimi, SM. Moattar-Hosseini[8]developed two heuristic approaches, including GA and an optimal enumeration method (OEM), to solve a new model of common cycle multi-product lot-sizing and scheduling problem in deterministic flexible flow shops with a finite planning horizon and limited intermediate buffers. The objective minimizes the sum of setup cost, inventory holding costs, and number of cycles. F. Riane, A. Artiba, S. Iassinovski [16] paid to the interaction between loading and scheduling problem in HFS environment. M.R. Garey, D.S. Johnson, R. Sethi [17] shows the optimal allocation of resources to activities over time is known to be combinatorially complex.

The HFS problem is, in most cases, NP-hard. For instance, HFS restricted to two processing stages, even in the case when one stage contains two machines and the other one a single machine, is NP-hard, after the results of J. N. D. Gupta [1]. Moreover, the special case where there is a single machine per stage, known as the flow shop, and the case where there is a single stage with several machines, known as the parallel machines environment, are also NP-hard, M. R. Garey and D. S. Johnson[2]. 
This study considers achieving optimal production volume in order to reduce production and inventory costs. Our main objective is to find a good allocation of jobs which generates a lower inventory level, a high plant efficiency and in which machines capacities are respected. The inventory level concerns in-process- stock and finished goods inventory. The efficiency of the plant means high machine utilization . This paper is organized as follows. In Section 2, we present the problem definition. In Section 3, we present the problem formulation. A solution procedure is introduced In Section 4. A to illustrate the propose approach, some examples are presented in Section 5.

\section{Hybrid Flow Shop}

A Hybrid Flow Shop (HFS) consists of series of production stages, each of which has several machines operating in parallel, that can be identical, uniform or unrelated. Some stages may have only one machine, but at least one stage must have multiple machines. The flow of jobs through the shop is unidirectional. Each job is processed by one machine in each stage and it must go through one or more stage (see fig.1)

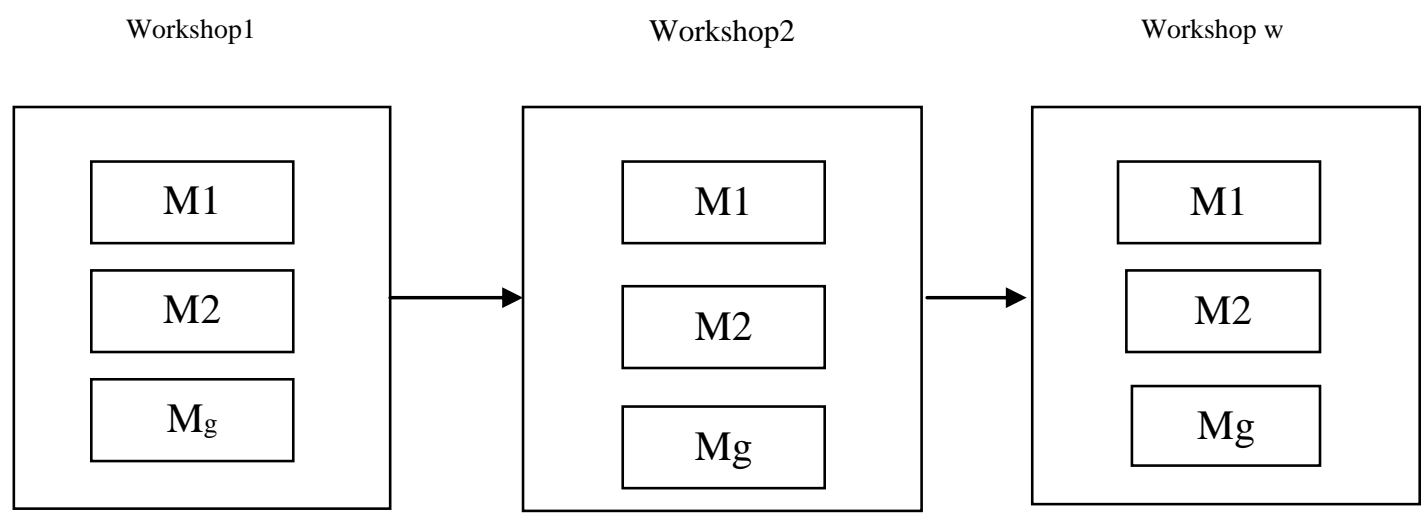

Fig.1.A structure of a hybrid flow shop organization

A job consists of several operations to be performed by none, one or more machines on each stage. The $\mathrm{i}$-th operation of a products (jobs) $i_{-}\{i / i=1,2, \ldots, n\}$ to be performed at the w-th workshop that Each workshop consists of $g \mathrm{w}$ centers of mg identical machines, requires units of time and can start only after the completion of the previous operation from the operation sequence of this job. Between the various stages of an hybrid flow shop environment there can be a definite amount of space, called buffer. This is a location in where the workflow can wait to be worked in the next stage. In the standard problem all jobs and machines are available at time zero, machines at a given stage are identical, any machine can process only one operation at a time and any job can be processed by only one machine at a time; setup times are negligible, preemption is not allowed, 
the capacity of buffers between stages is unlimited and problem data is deterministic and know in advance. A flow diagram of production planning and scheduling system is given in fig.2. In this paper, we presented a new mathematical model for the allocation jobs to each machine in each stage to optimize production and inventory costs in production planning process.

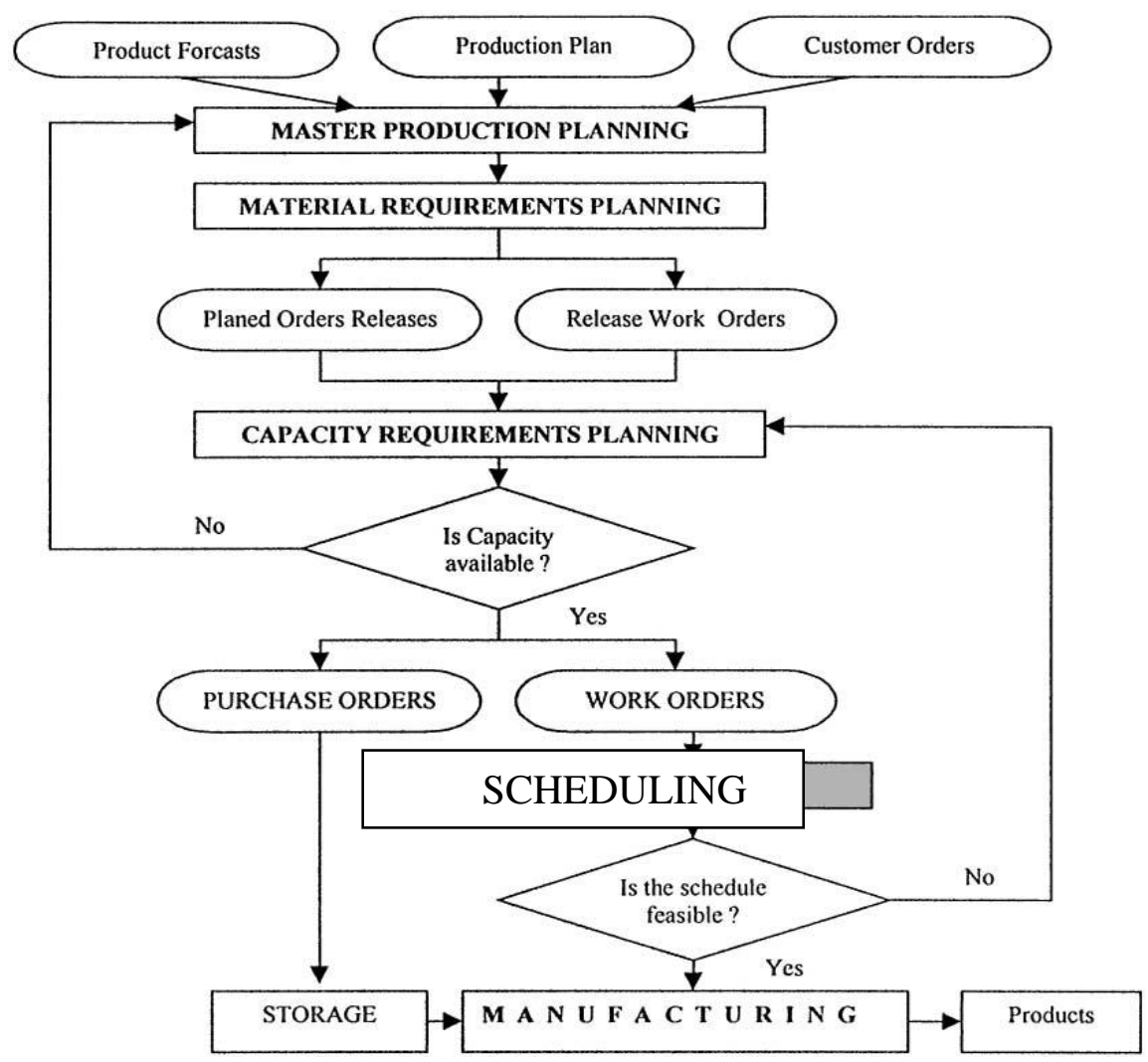

Fig.2. A flow diagram of production planning and scheduling system

\section{Mathematical Model}

The aims of this paper are minimize the:

- The total cost of production in t period.

- The total cost of inventory in the t period.

- The total cost of external production in the t period, and model assumptions are:

- The available time for each workshop is determined.

- The cost of internal production of products in each workshop is constant and deterministic.

- The cost of external production of products $\mathrm{i}$ is constant and deterministic. 
- Demand is constant and deterministic.

- Initial inventory level of products is determined.

- Available space for holding products for the period is specified.

Notations used for the problem formulation are as follow:

i :Product Type

$\mathrm{j}$ :workshop type

$\mathrm{m}$ :The number of machines in each workshop

$t:$ The number of time periods.

$\mathrm{X}_{\mathrm{ijmt}}$ : Amount of producing product $\mathrm{i}$ th in workshop $\mathrm{j}$ th with machine $(\mathrm{m})$ in period $\mathrm{t}$

$\mathrm{Z}_{\mathrm{it}}$ :The quantity of external product $\mathrm{i}$ at period $\mathrm{t}$

$\mathrm{Y}_{\mathrm{it}}$ :The stock level of product $\mathrm{i}$ by the end of period $\mathrm{t}$.

$\mathrm{C}_{\mathrm{ijmt}}$ : Cost of producing product $\mathrm{i}$ th in workshop $\mathrm{j}$ th with machine $(\mathrm{m})$ in period $\mathrm{t}$.

$C Z_{i t}$ :The cost of external product $\mathrm{i}$ at period $\mathrm{t}$.

$C Y_{i t}$ :The inventory holding cost of product $\mathrm{i}$

$t_{i j m t}$ : Time of producing product $i$ th in workshop $j$ th with machine $(m)$ in period $t$.

$\mathrm{p}_{\mathrm{jmt}}$ : Time available for machine $\mathrm{m}$ in the workshop $\mathrm{j}$

$\mathrm{D}_{\mathrm{it}}$ : The demand of product $\mathrm{i}$ in period $\mathrm{t}$.

fi : Available space for product i for stock .

The problem is formulated by:

$$
\begin{aligned}
& \min z=\sum_{t} \sum_{j} \sum_{m} \sum_{i} C_{i j m t} * X_{i j m t}+\sum_{t} \sum_{i} C Z_{i t} * Z_{i t}+\sum_{t} \sum_{i} C Y_{i t} * Y_{i t} \\
& Y_{i \mathrm{O}}=Y_{i} \quad \forall i \\
& \sum_{i} t_{i j m t} * X_{i j m t} \leq P_{i j t} \forall m, j, t \\
& \sum_{t} \sum_{i} X_{i j m t}+Y_{i 0}+Z_{i t}+Y_{i t-1} \geq D_{i t} \forall i, t \\
& \sum_{t} \sum_{i} Y_{i t} \leq F \\
& x_{i j m t} \geq 0 \quad Y_{i t} \geq 0, Z_{i t} \geq 0
\end{aligned}
$$

The objective function (1) minimizes the sum of internal production, external procurement, and inventory carrying costs. Equation (2) shows the initial inventory level at the time of beginning producing. Equation (3) shows the available time for each machine in each work station in any time period. Equation (4) shows minimal production level to meet the demands at any time period for each product. Equation (5) shows the maximum available space for keeping the inventory at end of each production period. 


\section{Genetic Algorithms for Problem Solving}

In this paper, the genetic algorithm is used in optimization process. The concept of GA was developed by Holland and his colleagues in the 1960s and 1970s [18]. The ability of GA to simultaneously search different regions of a solution space makes it possible to find a diverse set of solutions for difficult problems with nonconvex, discontinuous, and multi-modal solutions spaces.

\subsection{Chromosome structure}

In this paper, the chromosomes shows the quantity of job that allocated to each machine in each stage. At first, variables are encoded into binary threads. If variable $u_{j}$ is in $\left[a_{j}, b_{j}\right]$, then number of genes are repeat shown as $w_{j}$ and are calculated through equation (6).

$$
\stackrel{w j-1}{2} \leq\left(b_{j}-a_{j}\right) \times 10 \leq 2^{+k}-1
$$

That $k$ is the accuracy of variable $\mathrm{k}$.To convert a binary string to real numbers, we used equation 7.

$$
u_{j}=a_{j}+\text { decimal (substring) } \times \frac{b_{j}-a_{j}}{{ }_{2-1}^{w j}}
$$

For example, let two variables $u_{1}, u_{2}$ regarding range $[-3,12.1]$ and $[4.1,5.1]$. If $\mathrm{k}=3$ then:

$$
\begin{gathered}
\text { For } u_{1}: 2^{2} \leq\left(12.1-(-3) \times 1^{3} \leq 2^{18}-1 \rightarrow w_{1}=18\right. \\
\text { For } u_{2}: 2^{14} \leq(5.8-4.1) \times 10^{3} \leq 2-1 \rightarrow w_{2}=15
\end{gathered}
$$

As a result,the total chromosome length is 33 bits.

Genetic algorithm starts with a random initial population. The population is normally randomly initialized. The number of chromosomes inside the population is also need to be decided, because the number of the solutions determines the speed of the optimization and the accuracy of the solution found. If too many solutions are generated in the population, then longer duration of time is needed to find the fittest optimization. But, if the number of the solutions is too little or small, the genetic algorithm may face the problem of finding the fittest optimizations. 


\subsection{Fitness functions and selection mechanism}

The selection mechanism is one of the main search components in evolutionary algorithms. Since the solution space is very big, this study used normalizing technique to select parents to produce next generation. Equation 8 and 9 shows standard deviation of each generation and standard fitness function of each chromosome in each generation, respectively.

$$
\begin{aligned}
\sigma_{\mathrm{g}} & =\left(\frac{\sum_{\mathrm{i}=1}^{\mathrm{n}}\left(\mathrm{fit}_{(\mathrm{i})}-\mathbf{m}_{\mathrm{g}}\right)^{2}}{\mathbf{n}}\right)^{1 / 2} \\
\mathbf{z}_{\mathbf{i}}^{\mathrm{g}} & =\frac{\text { Fit(i) }-\mathbf{m}_{\mathrm{g}}}{\sigma_{\mathrm{g}}}
\end{aligned}
$$

Which $6_{\mathrm{g}}$, mg are mean and standard deviation of fitness functions in generation $g$ th and fit (i) is fitness function of chromosome $i$ th in generation ( $g$ ) th. After calculating the $\mathbf{z}_{\mathbf{i}}^{\mathbf{g}}$,if $\mathbf{z}_{\mathbf{i}}^{\mathrm{g}} \leq \mathbf{O}$,we selected chromosome $\mathrm{i}$ for next generation Because the problem goal is minimizing .(see Table.1).

Table.1. Mean and variance in generation $g$ and $g+1$

\begin{tabular}{|c|c|c|}
\hline \multicolumn{3}{|c|}{ generation $g+1$} \\
\hline $\begin{array}{l}\text { Normalized } \\
\text { fitness }\end{array}$ & Fit & Chromosome \\
\hline$z_{1}^{(g+1)}$ & $f_{1}^{g+1}$ & $u_{1}^{g+1}$ \\
\hline$z_{2}^{g+1}$ & $f_{2}^{g+1}$ & $u_{2}^{g+1}$ \\
\hline$\cdot$ & & $\cdot$ \\
$\cdot$ & & $\cdot$ \\
$\cdot$ & $f_{n}^{g+1}$ & $\cdot$ \\
\hline$z_{n}^{g+1}$ & & \begin{tabular}{c}
$u_{n}^{g+1}$ \\
\hline$m_{g} \cdot \sigma_{g}$
\end{tabular} \\
\hline
\end{tabular}

\begin{tabular}{|c|c|c|}
\hline \multicolumn{3}{|c|}{ generation $g$} \\
\hline $\begin{array}{c}\text { Normalized } \\
\text { fitness }\end{array}$ & Fit & Chromosome \\
\hline$z_{1}^{y}$ & $\mathrm{f}_{1}^{\mathrm{g}}$ & $u_{1}$ \\
\hline$z_{2}^{g}$ & $\mathrm{f}_{2}^{\mathrm{g}}$ & $u_{2}$ \\
\hline$\cdot$ & $\cdot$ & $\cdot$ \\
$\cdot$ & $\cdot$ & $\cdot$ \\
$\cdot$ & $\cdot$ & $u_{n}^{g}$ \\
\hline$z_{n}^{g}$ & $\mathrm{f}_{\mathrm{N}}^{\mathrm{g}}$ & $\begin{array}{c}\text { mean and } \\
\text { variance }\end{array}$ \\
\hline$m_{g}, \sigma_{g}$ & & \\
\hline
\end{tabular}

\subsection{Design the genetic operators}

GA use two operators to generate new solutions from existing ones: crossover and mutation. The crossover operator is the most important operator of GA. In crossover, generally two chromosomes, called parents, are combined together to form new chromosomes, called offspring. In this study for produce offspring from 
parents for entering the next generation, we used one-point crossover. For instance, in a intersection with two phases, two random chromosomes with feasible genes can be as follows:

$$
\begin{aligned}
& \text { Parent } 1=[0,1,1,1,0,0,1,0,0,1,1,0,0,0] \\
& \text { Parent } 2=[1,1,0,0,1,1,1,1,0,0,1,1,0,1]
\end{aligned}
$$

The offspring produced from these parents are as follows:

ONE-cut-point crossover with random points $(\mathrm{e} 1=9)$.

$$
\text { Offspring } 1=[0,1,1,1,0,0,1,0,0,0,1,1,0,1]
$$

Offspring $2=[1,1,0,0,1,1,1,1,0,1,1,0,0,0]$

The mutation operator used to be in order to create change, diversity and divergence in the population mutation operator in the classic mode, which means changing the values of two distinct genes(Mutations Replacement) together, or change the amount of the permissible scope of asingle gene(mutation changes). For instance, one point mutation with random points $(\mathrm{e} 1=6)$.

Parent $=[0,1,1,1,0,0,1,0,0,1,1,0,0,0]$

Offspring $=[0,1,1,1,0,1,1,0,0,1,1,0,0,0]$

The main steps of the Solution procedure are as follows:

Step 1: Set $g=1$. Randomly generate $\mathrm{N}$ solutions to form the first population, $\mathrm{p}_{\mathrm{g}}$.

Step 2: Evaluate the fitness of solutions in $\mathrm{p}_{\mathrm{g}}$.

Step 3: Selecting chromosome from the initial population with equation 8 and 9. Step 4: Crossover and mutation functions, reproducing the offspring and adding them to the initial population.

Step 5: $g=g+1$ and repeating steps 2-4 until a termination condition has been reached.

\section{Illustrative Example}

We consider the two-workshop of hybrid flowshop organization .Each workshop consist two different centers of machines (see fig.3). The model is programmed in the Microsoft Excel 2007 software using the Visual Basic Application (VBA). The problem data given in tables. 2, 3 and 4 is entered in the application software. 


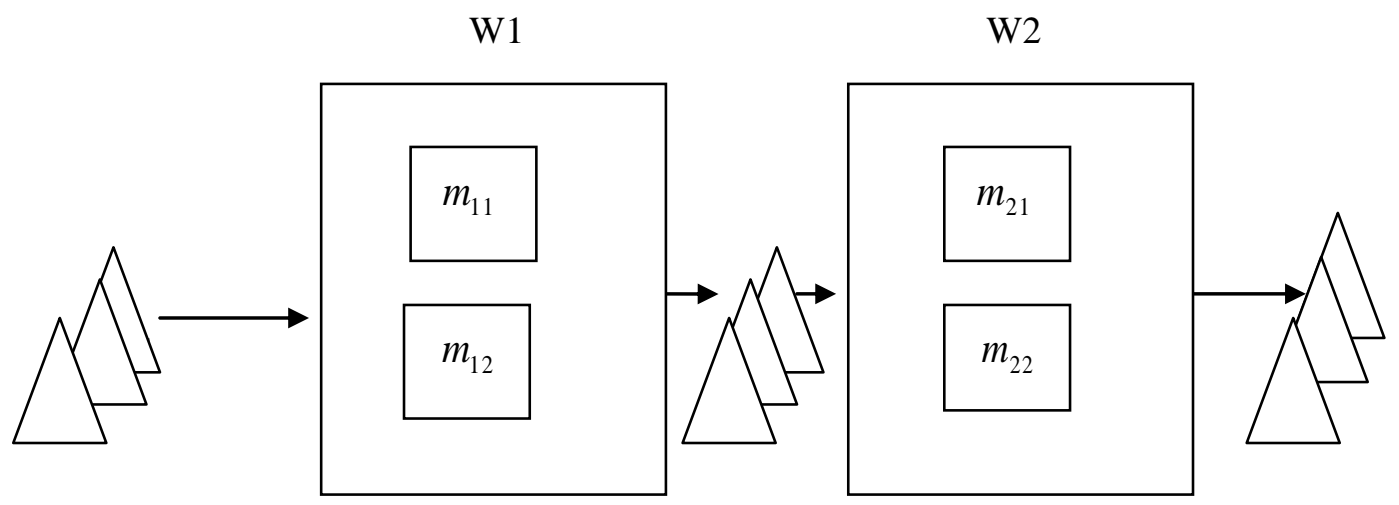

Fig.3.Description of the production process

Table.3.Internal production cost for each product

\begin{tabular}{|c|c|c|c|c|}
\hline Product & 1 & 2 & 3 & \\
\hline $\mathrm{m}_{11}$ & 300 & 800 & 150 & \\
\hline $\mathbf{m}_{12}$ & 300 & 800 & 150 & \\
\hline $\mathbf{m}_{21}$ & 800 & 400 & 500 & \\
\hline $\mathbf{m}_{22}$ & 800 & 400 & 500 & \\
\hline \multicolumn{5}{|c|}{$\begin{array}{l}\text { Table.4.External production cost for each } \\
\text { product }\end{array}$} \\
\hline Product & & 1 & 2 & 3 \\
\hline cost & & 2000 & 2000 & 2000 \\
\hline
\end{tabular}

Table.2.The processing times in the workshop

\begin{tabular}{lcccc}
\hline Product & 1 & 2 & 3 & $\begin{array}{c}\text { capacity } \\
\text { (min) }\end{array}$ \\
\hline $\mathrm{m}_{11}$ & 10 & 15 & 30 & 3200 \\
$\mathbf{m}_{12}$ & 6 & 20 & 25 & 3200 \\
$\mathbf{m}_{21}$ & 25 & 40 & 15 & 3200 \\
$\mathbf{m}_{22}$ & 30 & 10 & 20 & 3200
\end{tabular}

Table.7. The initial inventory level, demands, available space and holding cost for the jobs

\begin{tabular}{cccc}
\hline Product & 1 & 2 & 3 \\
\hline $\mathbf{y}_{\mathbf{i} 0}$ & 30 & 30 & 30 \\
$C Y_{i t}$ & 4000 & 4000 & 4000 \\
$\mathrm{fi}$ & 60 & 60 & 60 \\
$\mathrm{D}_{\text {it }}$ & 80 & 80 & 60 \\
\hline
\end{tabular}


The previous tables summarize the necessary data to be used in optimization process. Table 2 represents the processing times in the workshop 1and 2.Table 3 represents internal production cost for each product of the workshop 1and 2.table 4 represents external production cost for each product and table 5 represents the initial inventory level, demands, available space and holding cost. We assumed the production capacity of each center of each workshop equal to 3200 minutes .Our main objective is to find a optimal work order generates a lower inventory level,with lower internal and external production cost.

\subsection{Computing Results}

These algorithms are coded and implemented in Excel 2007 by the VBA and are ran on a Pentium 4 PC with CPU $2.8 \mathrm{GHz}$ and512 MB of RAM memory. Problem coded for both time periods $t=1$ (the feasible solution equal to $2^{\wedge} 96$ ) and $t=2$ (the feasible solution equal to $2^{\wedge} 196$ ) . (see table 8,9 and 10 )

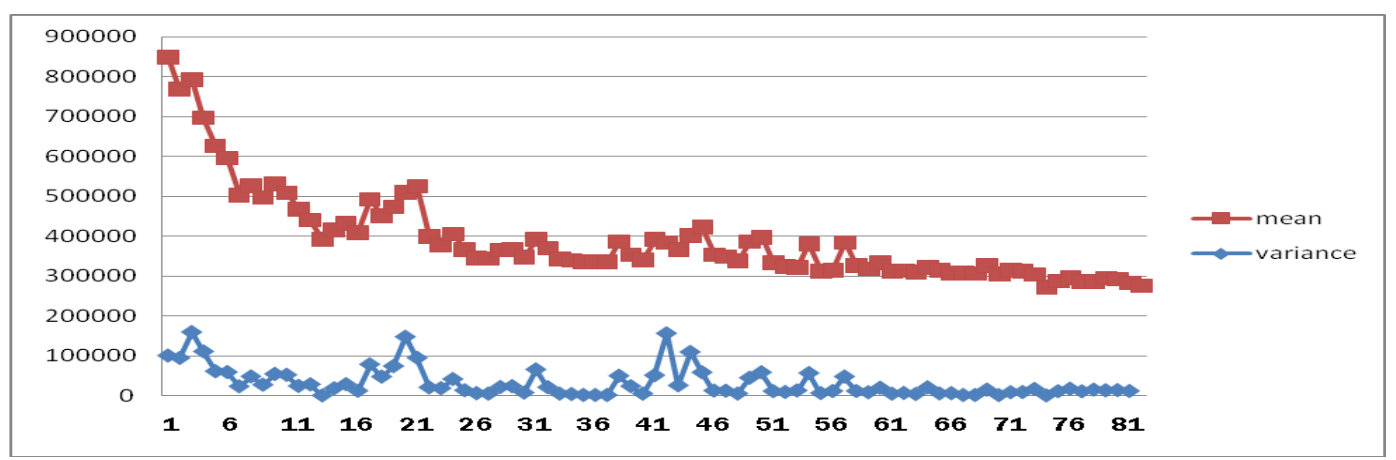

Fig.4. Mean and variance value with increasing the number of generations.

Reducing of fitness functions, variances in each repetition shows converging of the answers .due to the fact that reducing fitness functions variances show more similarities of chromosomes in each generation. Reduction of fitness functions mean in each repeat shows moving toward optimality.

Table.8.Sensitivity analysis for $\mathrm{t}=1$

\begin{tabular}{rrlrrrr}
\hline Generation & population & dimensions & $\begin{array}{c}\text { objective } \\
\text { function }\end{array}$ & Time(s) & Mean & Variance \\
\hline 400 & 100 & $18^{*} 10$ & 399907 & 107 & 631475 & 75266 \\
400 & 200 & $18^{*} 10$ & 384308 & 205 & 526948 & 74599 \\
400 & 300 & $18^{*} 10$ & 331062 & 275 & 512208 & 71008 \\
400 & 400 & $18^{*} 10$ & 330103 & 390 & 510001 & 70521 \\
400 & 600 & $18^{*} 10$ & 290520 & 410 & 400098 & 70498 \\
\hline
\end{tabular}


Table.9.Sensitivity analysis for $\mathrm{t}=2$

\begin{tabular}{rrrcrrr}
\hline Generation & population & dimensions & $\begin{array}{l}\text { objective } \\
\text { function }\end{array}$ & Time(s) & Mean & Variance \\
\hline 400 & 50 & $36 * 31$ & 2153494 & 130 & 2608258 & 223452 \\
400 & 200 & $36 * 31$ & 1630049 & 240 & 1847430 & 102148 \\
1000 & 400 & $36 * 31$ & 1578420 & 375 & 1728064 & 122608 \\
1000 & 800 & $36 * 31$ & 1617845 & 415 & 1775159 & 91647 \\
1500 & 900 & $36 * 31$ & 1419179 & 540 & 1710210 & 81962 \\
\hline
\end{tabular}

Table.10.Best solution with GA

\begin{tabular}{|c|c|c|c|c|c|c|c|c|c|c|c|c|}
\hline \multirow[t]{6}{*}{$t=1$} & $\mathrm{x} 1111$ & $\mathrm{x} 1211$ & $\mathrm{x} 1112$ & $\mathrm{x} 1212$ & $\mathrm{x} 2111$ & $\mathrm{x} 2211$ & $\mathrm{x} 2112$ & $\mathrm{x} 2212$ & x3111 & x3211 & x3112 & x3212 \\
\hline & 5 & 44 & & & 18 & 1 & & & 29 & 64 & & \\
\hline & x1121 & x 1221 & x1122 & x1222 & $\mathrm{x} 2121$ & x 2221 & x 2122 & x 2222 & x3121 & x3221 & x3122 & x3222 \\
\hline & 76 & 37 & & & 36 & 54 & & & 12 & 8 & & \\
\hline & y11 & 3 & y12 & & y21 & 1 & y22 & & y31 & 1 & y32 & \\
\hline & z11 & 0 & z12 & & z21 & 30 & $\mathrm{z} 22$ & & z31 & 19 & z32 & \\
\hline \multirow[t]{6}{*}{$t=2$} & x1111 & x1211 & x1112 & x1212 & x2111 & x2211 & $\mathrm{x} 2112$ & x2212 & x3111 & x3211 & x3112 & x3212 \\
\hline & 90 & 45 & 50 & 120 & 24 & 20 & 39 & 10 & 12 & 20 & 31 & 43 \\
\hline & x1121 & x1221 & x1122 & x1222 & x2121 & x 2221 & x2122 & x 2222 & x3121 & x3221 & x3122 & x3222 \\
\hline & 6 & 51 & 128 & 18 & 56 & 60 & 60 & 89 & 11 & 13 & 34 & 23 \\
\hline & y11 & 61 & y12 & 42 & y21 & 10 & y22 & 88 & y31 & 37 & y32 & 130 \\
\hline & z11 & 45 & z12 & 17 & $\mathrm{z} 21$ & 1 & $\mathrm{z} 22$ & 14 & z31 & 75 & z32 & 36 \\
\hline
\end{tabular}

\section{Conclusions}

In this paper, we proposed and optimization mathematical model of hybrid flow shop work order problem. . To solve the given problem, we applied the GA for solving the presented model. The high speed of the proposed algorithm and its quick convergence makes it desirable for large hybrid flow shop work order problem. Reduce the production costs of operational strategies and goals is the most productive institutions. For this purpose, we have optimized the production and inventory costs of each of the products produced in each period. However, as the problem is classified as NP-hard, it is very difficult to achieve optimal solutions using the integer programming(IP )model for larger problems. Therefore, we also introduce a genetic algorithm to efficiently find a good solution for the problem. 


\section{References}

[1] J. N. D. Gupta, Two-stage hybrid flow shop scheduling problem,Journal of the Operational Research Society, 39(4) (1988),359-364.

[2] M. R. Garey and D. S. Johnson, A guide to the theory of NP Completeness,San Francisco (1979).

[3] J. N. D. Gupta and E. A. Tunc,Scheduling a two-stage hybrid flowshop with separable setup and removal times. European Journal of Operational Research, 77(3) (1994),415-428.

[4] J. N. D. Gupta and E. A.Tunc,Schedules for a two-stage hybrid flowshop with parallel machines at the second stage, International Journal of Production Research, 29(7) (1991),1489-1502.

[5] J. N. D. Gupta,Two-stage, hybrid flow shop scheduling problem. Journal of the Operational Research Society, 39(4) (1988),359-364.

[6] K. Alaykýran, O. Engin, A. Döyen,Using ant colony optimization to solve hybrid flow shop scheduling problems, The international journal of advanced manufacturing technology,35 (2007), 541-550.

[7] M. Jenabi, S. M. T. Fatemi-Ghomi, S. A. Torabi, B. Karimi,Two hybrid meta-heuristics for the finite horizon ELSP in flexible flow lines with unrelated parallel processors, Applied Mathematics and Computation; 186(1) (2007), 230-45.

[8] B. Akrami, B. Karimi, SM. Moattar-Hosseini,Two metaheuristic methods for the common cycle economic lot sizing and scheduling in flexible flow shops with limited intermediate buffers the finite horizon case, Applied Mathematics and Computation,183 (2006),634-45.

[9] N. G. Hall, C. Srisk and arajah,A survey of machine scheduling problems with blocking and no-wait in process, Operations Research 44 (1996), 51025.

[10] M. S. Salvador,A solution to a special case of flow shop scheduling problems, in Symposium of the Theory of Scheduling and Applications, S.E. Elmaghraby (ed.), New York, Springer, 1973.

[11] C. Rajendran, D. Chaudhuri,Scheduling in n-job, m-stage flowshop with parallel processors to minimize makespan,International Journal of Production Economics 27 (1992)., 137-143.

[12] S. A. Brah, J. L. Hunsucker.(1991).Branch and bound algorithm for the flow shop with multiple processors. European Journal of Operational Research 51, 88-99.

[13] T. Sawik,A scheduling algorithm for flexible flow lines with limited intermediate buffers,Journal of Manufacturing Systems, 9 (1993), 127-38.

[14] T. Sawik,Scheduling flexible flow lines with no-process buffers,International Journal of Production Research, 33 (1995),1359-70. 
[15] T. Sawik,Mixed integer programming for scheduling surfacemount technology lines,International Journal of Production Research,39(1) (2001),3219-35.

[16] F. Riane, A. Artiba, S. Iassinovski,An integrated production planning and scheduling system for hybrid flowshop organizations, J. Production Economics 74 (2001), 33-48

[17] M.R. Garey, D.S. Johnson, R. Sethi,The complexity of flowshop and job shop scheduling, Mathematics of Operations Research ,1 (1976),117-129.

[18] Holland JH,Adaptation in natural and artificial systems, University of Michigan Press, 1975. 